\title{
CYBPET: a cylindrical PET system for breast imaging
}

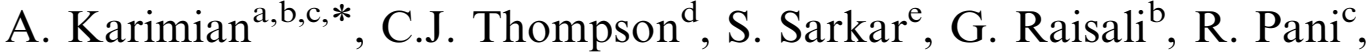 \\ H. Davilu ${ }^{a}$, D. Sardari ${ }^{\mathrm{a}}$ \\ a Amirkabir University of Technology, Tehran, Iran \\ ${ }^{\mathrm{b}}$ Nuclear Research Center for Agriculture and Medicine (NRCAM-AEOI), P.O. BOX. (31485-498), Karaj, Iran \\ ${ }^{\mathrm{c}}$ Department of Experimental Medicine and Pathology, University of Rome La Sapienza, Rome, Italy \\ ${ }^{\mathrm{d}}$ Montreal Neurological Institute, McGill University, Montreal QC, Canada \\ ${ }^{\mathrm{e}}$ Medical physics Department of Tehran University of Medical Sciences \& (RCSTIM), Tehran, Iran
}

Received 23 September 2004; received in revised form 7 January 2005; accepted 20 January 2005

Available online 28 March 2005

\begin{abstract}
We propose a Cylindrical Breast PET (CYBPET) system for breast imaging with patients in the prone position. An individual pendulous breast is covered by thin plastic to provide reduced pressure fixation and surrounded by the crystals inside the CYBPET ring. Each breast is imaged separately. The rest of the body is shielded properly to minimize the contribution of scattered photons from the other breast and the rest of the body. To compare the CYBPET with whole-body PET (WB-PET) the simulations of CYBPET and a WB-PET (GE-Advance) for a $10 \mathrm{~mm}$ tumor inside the breast with a lesion to background (breast) activity concentration of 6 to 1 were made. The noise effective count rate (NECR) of CYBPET is about twice that of WB-PET at activity concentrations less than $3.1 \mu \mathrm{Ci} / \mathrm{cc}$. The spatial resolution of CYBPET is better by $25 \%$ than the WB-PET.
\end{abstract}

(C) 2005 Elsevier B.V. All rights reserved.

PACS: 87.62. + n; 87.59. Vb

Keywords: Positron emission mammography; Positron emission tomography; Nuclear medicine instrumentation; Breast imaging; Breast cancer; PEM

\footnotetext{
*Corresponding author. Nuclear Research Center for Agriculture and Medicine (NRCAM-AEOI), P.O. BOX. (31485-498), Karaj, Iran. Fax. + 982614411106.

E-mail address: akarimian@nrcam.org
} (A. Karimian).

\section{Introduction}

Breast cancer represents the most frequent malignant disease in women and the second leading cause of cancer death in industrialized countries and in Iran. The disease is often curable when detected in early stages [1,2]. Therefore, early 
diagnosis which significantly improves the 5-year survival rate, is very important in the successful treatment [3]. Mammography and physical breast examination, both non-invasive techniques, provide the two most effective methods available for screening potential breast cancer patients. The main limitation of X-ray mammographic methods is their low specificity (not differentiating between benign and malignant lesions). This requires one of several invasive techniques such as core biopsies or lumpectomy to be performed in order to determine the stage or malignancy of the disease $[4,5]$. Each of these techniques is economically and psychologically burdensome, especially if these exams demonstrate they were not necessary. It has been shown that, because of the limited specificity of mammography for non-palpable cancers, between $70-90 \%$ of excisional biopsies performed on these kinds of tumors were unnecessary because they were benign [6].

Positron Emission Tomography (PET), using [F-18] fluoro-deoxyglocose (FDG) as the tracer and whole-body clinical scanners, has been shown to be highly sensitive and specific in functional studies of breast cancer and may be a cost-effective alternative to invasive procedures under certain circumstances [4]. However, conventional WBPET systems have several shortcomings for breast imaging related to the general purpose nature of these systems. (I) In WB-PET scanners, the detectors are typically $20-30 \mathrm{~cm}$ away from the breast or axilla and this reduces their sensitivity [7]. (II) The patient's torso is between radioactive sources in the breast cancers and the PET detectors for most views over a complete $360^{\circ}$ and therefore patient torso is the fundamental attenuator for breast images in WB-PET scanners. (III) Higher uptake in myocardium and liver than in breast cancers may contaminate breast cancer images because of Compton scattered events.

To overcome these limitations and improve the overall diagnostic quality of breast cancer images, a number of research groups, have worked on dedicated Gamma Cameras and PET systems for breast imaging [5-17]. Some of these dedicated systems such as the PEM-flex (PET Systems, Rockville, MD USA) [18] and SPEM [14] are used clinically at present. However, most of these dedicated systems, in spite of their advantages, have small field of view (FOV) and/or cannot work in tomography mode. Also some of them cannot be used for the patients under radiotherapy, or after breast surgery. Therefore, we propose the use of CYBPET system for breast imaging for patients in the prone position [19].

\section{Materials and methods}

The CYBPET consists of 6912 bismuth germanate (BGO) crystals $(3.0 \mathrm{~mm}$ transaxial by $5.0 \mathrm{~mm}$ axial by $20 \mathrm{~mm}$ radial) which are grouped in detector blocks of $8 \times 8$ crystals each, with 32 rings and a transaxial and axial FOV of $17.0 \mathrm{~cm}$ with a ring diameter of $22 \mathrm{~cm}$ (Fig. 1). A $20 \mathrm{~cm}$ port diameter (suitable for almost all adult female breast sizes) surrounds an individual pendulous breast which is under mildly reduced pressure within a thin flexible plastic membrane. A vacuum pump will fix the pendulous breast in the center of FOV. The rest of the body is shielded properly to minimize the contribution of scattered photons from the other breast and the rest of the body. For this reason the bed is covered by a $3 \mathrm{~mm}$ lead shield. In order to prevent the cross talk between the block detectors, each detector unit was separated from the adjacent one by lead and kovar. The crystals are coupled directly and viewed by avalanche photodiodes (APD). APDs have some advantages which make them attractive for high-resolution PET systems, such as, their high detection quantum efficiency (i.e. converting the scintillation light into electrons), their compact physical size which allows a high packing fraction of small discrete detector elements for highresolution imaging and potentially lower cost. However, APDs need strict control of both bias voltage and operating temperature. They also require individual fast, high gain, low-noise preamplifiers [13,20].

To assess a number of performance characteristics and breast imaging of this system with a currently available conventional WB-PET system (GE Advance, GEMS, Milwakee WI) whose performance characteristics were available to us, we used the Monte Carlo simulation programming 
package: "PETSIM" [21-23]. These programs simulate the source fluence, then the events which pass the collimator, and finally those rays which are detected in separate files all of which must coexist on one $2 \mathrm{~GB}$ disk. Each $\gamma$-ray requires

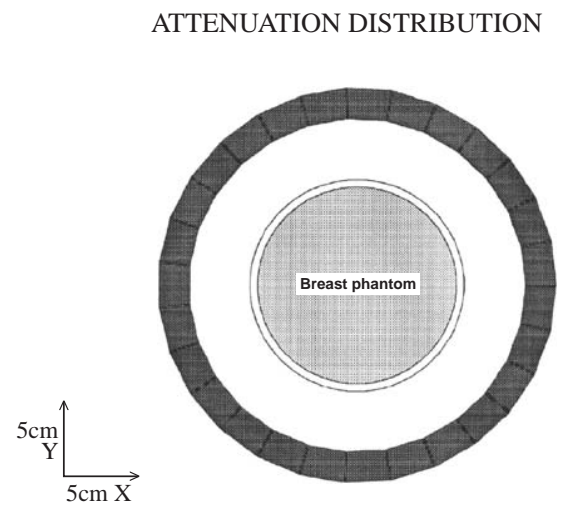

(a)

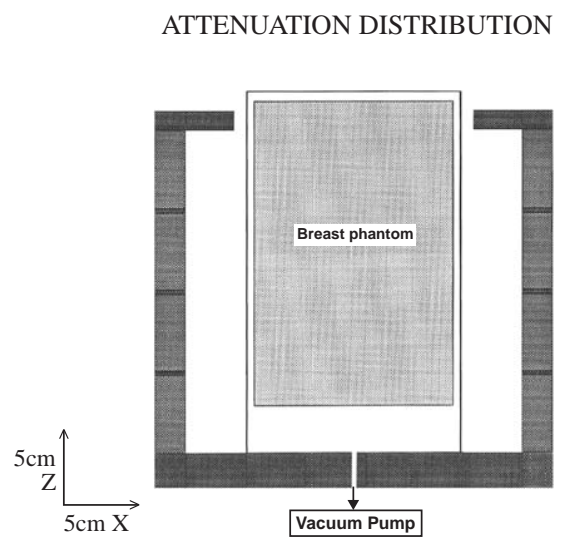

(b)
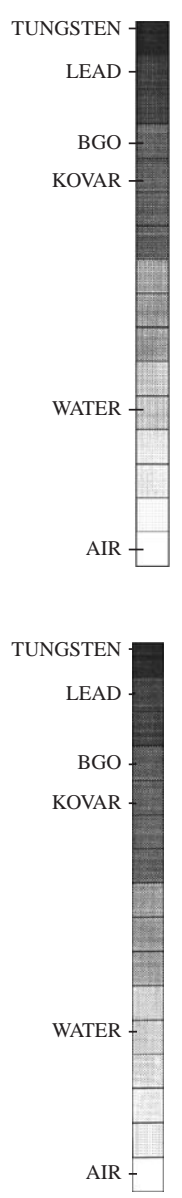

Fig. 1. Top (a) and front (b) views of CYBPET. The blackening is proportional to the linear attenuation coefficient of materials.
20 bytes of storage. The simulation data files consist of blocks containing $256 \gamma$-rays, or 5.12 Kbytes. Generally, in simulation programs, higher numbers of simulated counts give the better precision and signal to noise ratio and image quality because of lower statistical noise. Therefore, to reduce the effects of statistical on the output results of simulations in this research, the maximum number of output blocks which could be saved (i.e. 100,000 blocks) was used for $\gamma$-ray history file (GRH) of "phantom" program and for the other programs in PETSIM namely "collimator" and "detector" the GRH files were selected proportional to this maximum number of blocks.

The standard settings which were considered for CYBPET and WB-PET (GE-Advance) are as in Table 1.

The published data on the performance of PET scanners does not match the scanning geometry which is being proposed here. However, when the performance of new PET scanners is first reported, it is often done by reference to a standard phantom proposed by the National Electrical Manufacturers Association of the USA (NEMA). In order to calibrate the simulation results for breast imaging, we first simulated the performance of the WB-PET with a NEMA phantom. The NECR was estimated over a range of activity concentration and compared with published data from DeGrado et al. [21].

These two systems, CYBPET and GE-Advance WB-PET, were then assessed and compared with each other for breast imaging. For this purpose the breast was simulated by a cylinder $(13 \mathrm{~cm}$ diameter by $16 \mathrm{~cm}$ length). Lesions with various sizes can be assumed anywhere inside the breast. As an example a $10 \mathrm{~mm}$ cylindrical lesion was assumed very close to surface of the breast. This has been shown for CYBPET and WB-PET in the Fig. 2

Table 1

Standard settings for WB-PET and CYBPET

\begin{tabular}{|c|c|c|c|c|c|}
\hline \multirow[t]{2}{*}{ PET scanner } & \multirow[t]{2}{*}{ Coincidence window (ns) } & \multirow[t]{2}{*}{ Dead time $(\mu \mathrm{s})$} & \multirow[t]{2}{*}{ Energy resolution (\%) } & \multicolumn{2}{|c|}{ Energy discriminator $(\mathrm{keV})$} \\
\hline & & & & Lower level & Upper level \\
\hline WB-PET (GE-Advance) & 12.5 & 3.6 & 20 & 300 & 650 \\
\hline CYBPET & 12.5 & 3.6 & 20 & 400 & 650 \\
\hline
\end{tabular}




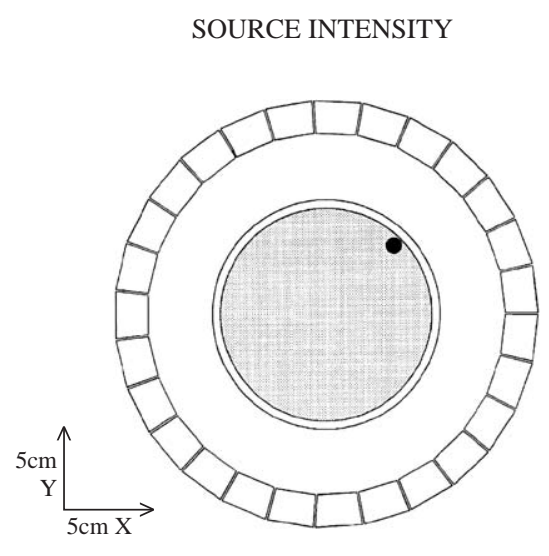

(a)

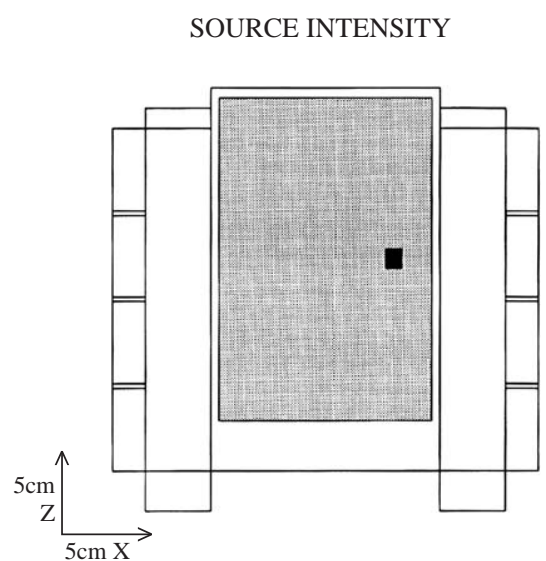

(b)

Fig. 2. Top (a) and front (b) views of CYBPET. A $10 \mathrm{~mm}$ lesion has been located very close to the skin of breast inside the breast phantom. The blackening is proportional to the source intensity.

and Fig. 3, respectively. This $10 \mathrm{~mm}$ lesion was located at radiuses of 5.9 and $16.8 \mathrm{~cm}$ from the geometric center of CYBPET and WB-PET, respectively. The center of lesion was $6 \mathrm{~mm}$ from the breast skin in both systems. A lesion to soft tissue (healthy breast) activity concentration of 6 to 1 was used in our simulation. This is the same contrast found by other authors, for example: Murthy et al. [3], Freifelder and Karp [5] and White et al. [11].

The body was simulated by six nested cylinders, of which the biggest, has the dimensions of $(35 \mathrm{~cm}$
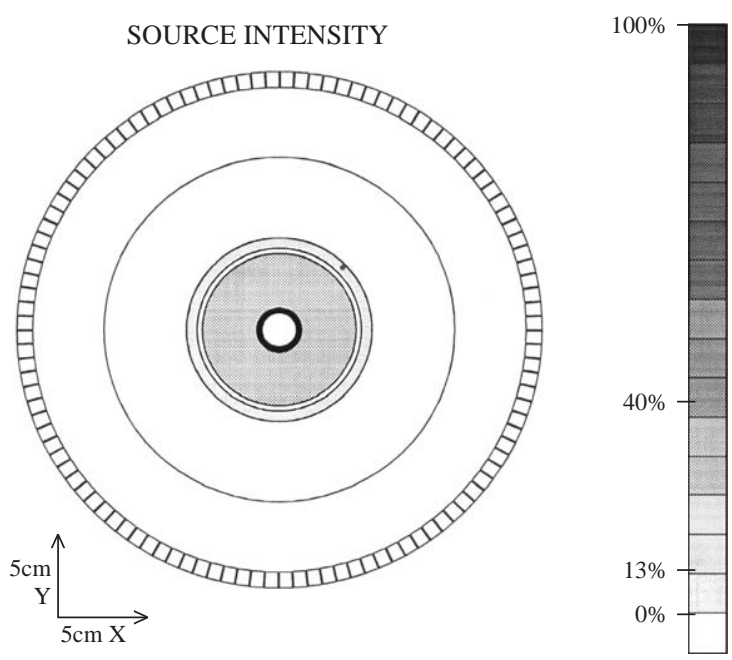

Fig. 3. Activity distribution inside the body phantom with a $10 \mathrm{~mm}$ lesion very close to the skin of breast in the WB-PET system. The blackening is proportional to the source intensity.

diameter by $24 \mathrm{~cm}$ length) by taking account the effects of scatter and accumulated activity related to the thorax and its tissues such as heart. The myocardium was modeled as a cylindrical shell with inner and outer radius of 3.26 and $4.26 \mathrm{~cm}$, respectively, with the approximate volume of $200 \mathrm{cc}$. The activity concentrations in the heart, lesion, lungs and all other healthy soft tissues are in the ratio of: $(15: 6: 2: 1)$ in the body phantom. ${ }^{1}$ The breast imaging simulation with a lesion size less than $10 \mathrm{~mm}$ with the mentioned lesion to background (breast) activity concentration was impossible with the WB-PET system in the simulation study, because in this case, less than $0.01 \%$ of annihilations would have occurred in the lesion and the lesion would be rejected by the simulation program.

\footnotetext{
${ }^{1}$ The activity concentration of (15:1:1) for (Heart: Breast (soft tissue): chest) has been used by Singh et al. [15] and also activity concentration of (15:1) for (Heart: Breast (soft tissue)) has been used by Wang et al. [16] for Tc-99 m (Sestamibi) distribution. Because both of FDG and Tc-99 m (Sestamibi) are the normal choices for heart and breast imaging and the ratio of uptake is used, so the uptake ratio of 15 has been used in our study too. Also there are tissues with high uptake (liver) and some large volumes such as lungs inside the thorax so the average uptake ratio of two was used for lungs which take a considerable volume of thorax.
} 
In order to estimate the spatial resolution of the new scanner, CYBPET, relative to a WB-PET system in the $3 \mathrm{D}$ mode, we again used PETSIM. The resolution module in PETSIM works best when a uniform source is used, as opposed to several very small sources which would be most accurate in a real experiment [22]. The program knows the location of each positron emitting nucleus, and can estimate the normal distance between the line of response corresponding to that joining the centers of the two crystals in which the annihilation photons interact and the location of the nucleus. In this case we assumed ${ }^{18} \mathrm{~F}$ positrons, non-collinearity of $0.5^{\circ}$, and divided the field into five radial bins. For this purpose the air cylinders with diameters of 17 and $50 \mathrm{~cm}$ were simulated inside the CYBPET and WB-PET respectively to cover the entire imaging FOV of both systems. The mean radii of the bins were: $0.85,2.55,4.25,5.95,7.65 \mathrm{~cm}$ for CYBPET and $2.5,7.5,12.5,17.5,22.5 \mathrm{~cm}$ for WB-PET. The resolution measurements were done for both systems by including the effects of both positron range and annihilation photon non-collinearity. We also estimated the advantages of radial subdivision of the crystals into three separate equal layers in both CYBPET and WB-PET scanners. This ability promises to be more precise for the measurements as mentioned in the Ref. [23].

Finally, the CYBPET was simulated by changing the crystal from $\mathrm{BGO}$ to lutetium oxyorthosilicate (LSO) with the same dimensions as BGO, to assess the benefits of using faster crystals in the CYBPET. The potential advantage of LSO compared to BGO is its much higher light output (approximately a factor of four) and shorter (about a factor of seven) primary pulse decay constant (40 versus $300 \mathrm{~ns}$ ). A potentially negative quality of LSO is the presence of Lu-176, which emits electrons (average emission energies of $50 \mathrm{keV}$ ) and a number of $\gamma$-rays (energies range from 88 to $401 \mathrm{keV}$ ) [24,25].

The coincidence resolving and signal integration times assumed for BGO were 12.5 and $300 \mathrm{~ns}$ and for LSO from 1 to 6 and $50 \mathrm{~ns}$, respectively. For this study, a lucite cylinder phantom with the dimensions of (input diameter $=14 \mathrm{~cm}$, length $=14 \mathrm{~cm}$ ) was used because of the port diameter of CYBPET.

\section{Results}

In Fig. 4, for the whole-body scanner in the 3D mode, the NECR curve from the simulation has been plotted. It is compared with the experimental values published by DeGrado et al. [21]. These curves show a maximum of 202 (159) kcps at the same activity concentration, i.e. $0.65 \mu \mathrm{Ci} / \mathrm{cc}$. This result shows a relative difference of about $27 \%$ for NECR (max) between simulation and experimental study. This discrepancy is probably due to the differences in actual discriminator settings and energy resolution which are present in individual detectors in a real PET scanner.
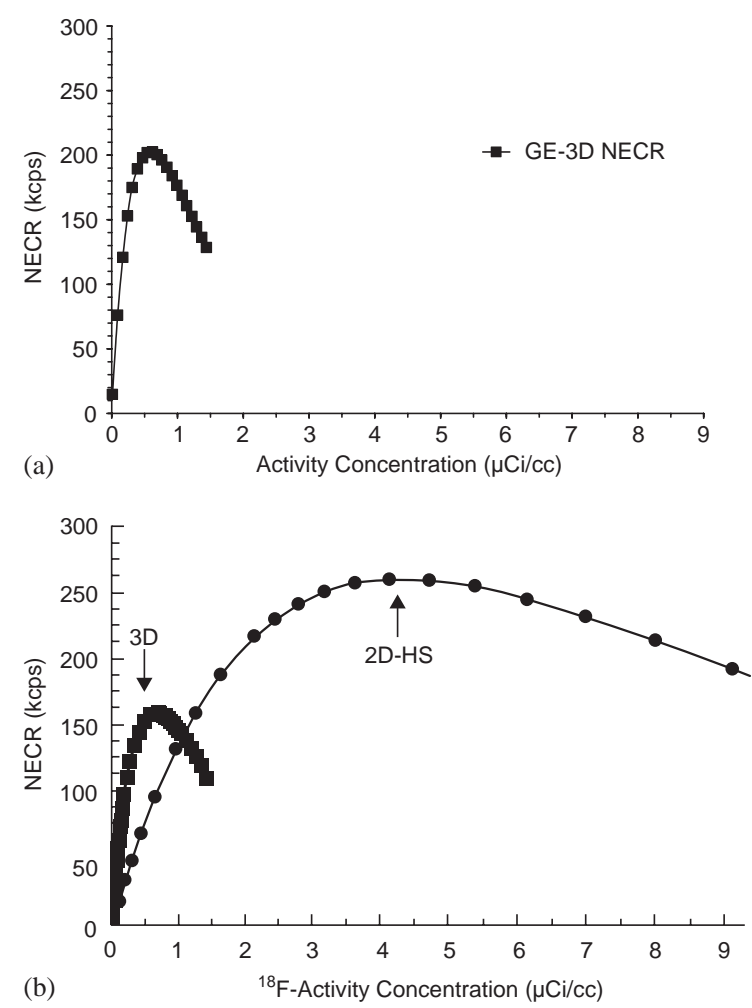

Fig. 4. NECR curve for WB-PET (GE-Advance) by using a standard lucite cylindrical phantom (20 cm ID, $18.5 \mathrm{~cm}$ length), Simulation result (a) and experimental results for $3 \mathrm{D}$ and 2D (High Sensitivity) modes reproduced from DeGrado et al. [21] (b). 


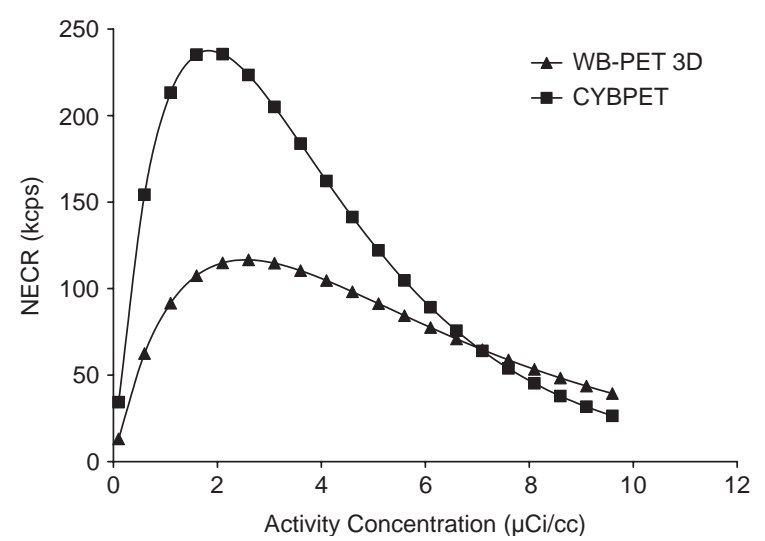

Fig. 5. NECR curves for breast imaging with a $10 \mathrm{~mm}$ diameter lesion located very close to the skin of breast for both WB-PET and CYBPET systems.

The NECR curves for breast imaging with a $10 \mathrm{~mm}$ diameter lesion located very close to the skin of breast are presented in the Fig. 5 for both WB-PET and CYBPET systems. This position of lesion is the best case for WB-PET.

Comparison of systems-averaged NECR curves shows this index to be higher for CYBPET than for WB-PET at radioactivity concentrations under $7.1 \mu \mathrm{Ci} / \mathrm{cc}$. At radioactivity concentrations below $3.1 \mu \mathrm{Ci} / \mathrm{cc}$, the CYBPET NECR is approximately twice as high than WB-PET. The maximum NECR for CYBPET (WB-PET) are 237 (117) kcps at a radioactivity concentration of $1.8(2.6) \mu \mathrm{Ci} / \mathrm{cc}$ which indicates the NECR (max) of CYBPET is occurred in a less activity concentration than WB-PET.

Furthermore, the coincidence efficiency for unscattered $\gamma$-ray pairs of both systems has been assessed by PETSIM. The efficiency was simulated as 692 and $387(\mathrm{kcps} / \mu \mathrm{Ci} / \mathrm{cc})$ for CYBPET and WB-PET systems, respectively.

The CYBPET was also simulated in the detection of lesions with different dimensions from 5 to $20 \mathrm{~mm}$ inside the breast. These lesions were located both in the center of breast and very close to the skin of breast in a distance of $1 \mathrm{~mm}$ from the skin. The relative coincidence efficiency and maximum NECR, are presented in Table 2 for lesions in the center and near the edge of the breast as a function of lesion size. The results in this table show the sensitivity and NECR are not highly dependent on
Table 2

The relative sensitivity and maximum NECR for lesions in the center and near to breast skin as a function of lesion size

\begin{tabular}{lll}
\hline $\begin{array}{l}\text { Lesion size } \\
(\mathrm{mm})\end{array}$ & $\begin{array}{l}\text { Sensitivity ratio of } \\
\text { Lesion (close to skin/ } \\
\text { in the center) }\end{array}$ & $\begin{array}{l}\text { NECR (max) ratio } \\
\text { of lesion (close to } \\
\text { skin/in the center) }\end{array}$ \\
\hline 5 & 1.00 & 1.00 \\
8 & 0.99 & 0.99 \\
10 & 0.99 & 0.99 \\
12 & 1.00 & 1.00 \\
15 & 1.00 & 1.00 \\
17 & 1.00 & 1.00 \\
20 & 1.00 & 1.01 \\
\hline
\end{tabular}

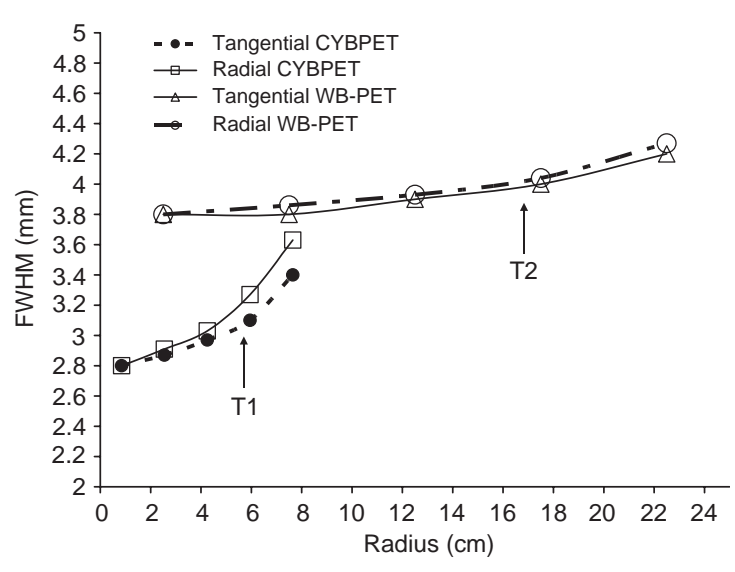

Fig. 6. Radial and tangential components of spatial resolution for CYBPET and WB-PET by simulation. T1 and T2 shows the position of lesions in the (Figs. 2 and 3). Each point represents the average simulated FWHM in a region within \pm 0.85 for CYBPET and $\pm 2.5 \mathrm{~cm}$ for WB-PET

the position of the lesion inside the breast for CYBPET scanner. This suggests that in this scanner attenuation correction may not be necessary for lesion detection.

Assessment of resolution for WB-PET and CYBPET is presented in Fig. 6. The curves show the minimum resolution (FWHM) for both CYBPET and WB-PET as 2.8 and $3.8 \mathrm{~mm}$, respectively. The locations of the simulated lesions (from the results in Figs. 2 and 3) have been identified with arrows (T1, T2) in Fig. 6. The marked points show the FWHMs of 3.2 and $4 \mathrm{~mm}$ for CYBPET and WB-PET, respectively. The resolution curves show the superior spatial 


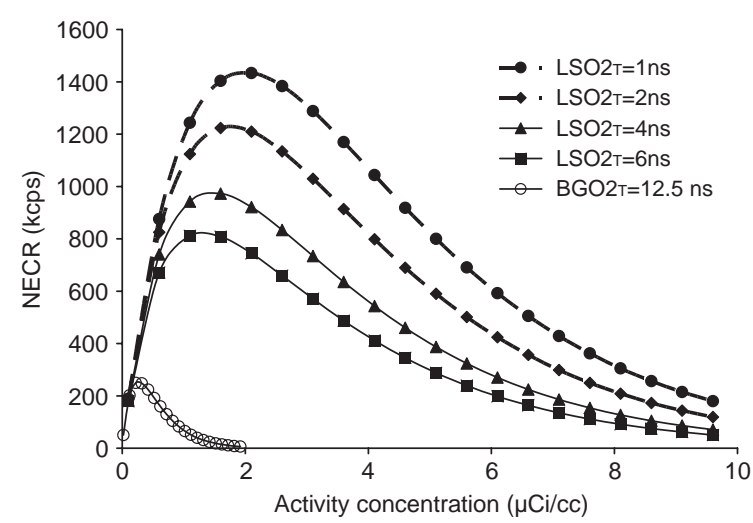

Fig. 7. NECR of CYBPET with BGO (dead time 3.6 $\mu \mathrm{s}$ ) and LSO (dead time $0.5 \mu \mathrm{s}$ ) crystals with different resolving times by a cylindrical phantom.

resolution of CYBPET against WB-PET over its full FOV. For instance, the resolution of CYBPET is about $80 \%$ of WB-PET for about $70 \%$ of its FOV.

The benefits of the substitution of LSO instead of BGO crystals are shown in the Fig. 7.

These curves show, the NECR (max) will increase from $250 \mathrm{kcps}$ for $\mathrm{BGO}$ with resolving time of $12.5 \mathrm{~ns}$ and integration time of $300 \mathrm{~ns}$ to $821,975,1228,1435 \mathrm{kcps}$ for LSO with resolving times of $6,4,2,1 \mathrm{~ns}$, respectively, and an integration time of $50 \mathrm{~ns}$. The dead time was assumed 3.6 and $0.5 \mu$ s for BGO and LSO, respectively.

The full benefits of the substitution of BGO by LSO are not considered in this simulation. PETSIM can track until the $\gamma$-ray detection, but not the propagation of the light within the crystals. Since there is much more light available from LSO, the crystals in each block are better identified, so fewer decoding errors can be expected. Also because the LLD of CYBPET was chosen equal to $400 \mathrm{keV}$ the additional emitted $\gamma$-rays due to existence of Lu-176, i.e. $\gamma$ rays with energy from 88 to $401 \mathrm{keV}$, were ignored in our study.

\section{Conclusion}

We have shown that new scanner, CYBPET, has the ability to yield improved image quality for breast imaging over WB-PET systems because of better resolution, sensitivity and NECR. The NECR is much better at low activity concentration. For example, the NECR of CYBPET was about twice that of the WB-PET at an activity concentration of less than $3.1 \mu \mathrm{Ci} / \mathrm{cc}$ for a $10 \mathrm{~mm}$ lesion. It has been shown that $[26,27]$ in the breast and breast tumors there is not high blood flow and metabolism and therefore low activity concentration, so a relative high NECR in the low radioactivity concentration is very important for detection of small tumors. Also this system does not need attenuation correction for breast imaging therefore, the scan time and total patient dose will be reduced accordingly. Furthermore, minimization of the volume and number of detectors and related electronics reduces the price of the system and the cost of a patient scan. Also, in comparing CYBPET with other PEM systems, the two most conventional PEM systems have two parallel planes and rectangular geometries. In comparison the CYBPET with two parallel planes, the CYBPET has more sensitivity because of its whole $360^{\circ}$ coverage that will reduce the scan time (or increase patient frequency) and or the injected dose which can compensate the increased price of CYBPET in a duration of time. Furthermore, it has been shown that [28] the images from the parallel plane PEM will suffer from some blurring or degraded spatial resolution, because of the large gap in the angular coverage.

In comparison to the proposed system with the rectangular geometry, both have full coverage of angle, and CYBPET would have a little initial higher cost because of more detectors, but it has suitable FOV for whole breast imaging in a reduced time to cover most of the female population and does not have a dead zone. Also the prone position for the patient is more comfortable than the mammography scan position. This is important since PEM scans take more time than mammography. It is quite uncomfortable for the patient to be in a mammography position for a considerable time. Furthermore, because the CYBPET has similar geometry to that of WB-PET, it has the potential of using similar image reconstruction techniques and as those used in WBPET. This should provide more accurate imaging 
than the other dedicated instruments for PEM because of more than 15 years experience behind these methods. Furthermore, we believe that clinical diagnosis is more difficult as tumor has irregular shape and its position is usually unknown in an incompletely uniform background of breast. At present the new system has not yet been constructed, so it is not possible to show its optimal role completely. However, the simulation results are promising, and we believe the detector's geometry is very favorable in this application.

\section{Acknowledgment}

The support of the "ICTP program for Training and Research in Italian Laboratories, Trieste, Italy" is acknowledged and appreciated in this work by Alireza Karimian. Also we would thank Dr. Paolo Bennati, Dr. Maria Nerina Cinti and Dr. Margherita Betti from the Department of Experimental Medicine and Pathology, University of Rome La Sapienza, Rome, Italy and Mr. Behrang Mossalla from the Nuclear Research Center for Agriculture and Medicine (NRCAMAEOI), Karaj, Iran.

\section{References}

[1] N. Avril, M. Schelling, J. Dose, W.A. Weber, M. Schwaiger, Utility of PET in Breast Cancer, Clin. Positron Imaging 2 (5) (1999) 261.

[2] Health Ministry of Irans' Report, 1998.

[3] K. Murthy, M. Aznar, C.J. Thompson, R. Lisbona, A. Loutfi, J.H. Gagnon, Preliminary clinical evaluation of an instrument for positron emission mammography (PEM-I), J. Nucl. Med. 41 (12) (2000) 1851.

[4] N.K. Doshi, R.W. Silverman, Y. Shao, S.R. Cherry, maxPET: a dedicated mammary and axillary region PET imaging system for breast cancer, IEEE Trans. Nucl. Sci 48 (3) (2001) 811.

[5] R. Freifelder, J.S. Karp, Dedicated PET scanners for breast imaging, Phys. Med. Biol 42 (1997) 2463.

[6] C.J. Thompson, K. Murthy, Y. Picard, I.N. Weinberg, R. Mako, Positron emission mammography (PEM): a promising technique for detecting breast cancer, IEEE Trans. Nucl. Sci. 42 (4) (1995) 1012.

[7] N.K. Doshi, Y. Shao, R.W. Silverman, S.R. Cherry, Design and evaluation of an LSO PET detector for breast cancer imaging, Med. Phys. 27 (7) (2000) 1535.
[8] W.W. Moses, T.F. Budinger, R.H. Huesman, S.E. Derenzo, PET camera designs for imaging breast cancer and axillary node involvement, J. Nucl. Med. 36 (1995) 69P.

[9] I. Weinberg, S. Majewski, et al., Preliminary results for positron emission mammography: real time functional breast imaging in a conventional mammography gantry, Eur J. Nucl. Med. 23 (1996) 804.

[10] W. Worstell, O. Johnson, H. Kudrolli, V. Zavarzin, First results with high-resolution PET detector modules using wavelength-shifting fibers, IEEE Trans. Nucl. Sci. 45 (6) (1998) 2993.

[11] L.L. White, R.J. Ott, M.A. Flower, K. Erlandsson, D.M. Duxbury, P. Bruyndonckx, S. Tavernier, Preliminary Evaluation of High Resolution Positron Emission Tomography for breast imaging, IEEE Conf. Rec. (2001).

[12] M.F. Smith, R.R. Raylman, S. Majewski, Positron emission mammography with multiple angle acquisition, IEEE Conf. Rec. (2002).

[13] P. Lecoq, J. Varela, Clear-PEM, a dedicated PET camera for mammography, Nucl. Instr. and Meth. Phys. Res. A 486 (2002) 1.

[14] R. Pani, G. De Vincentis, F. Scopinaro, R. Pellegrini, A. Soluri, Dedicated Gamma Camera for Single Photon Emission Mammography (SPEM)., IEEE Trans. Nucl. Sci. 45 (6) (1998) 3127.

[15] M. Singh, E. Mumcuoglu, Design of a CZT-based breast SPECT system, IEEE Trans. Nucl. Sci. 45 (3) (1998) 1158.

[16] H. Wang, C. Scarfone, K.L. Greer, R.E. Coleman, R.J. Jaszczak, Prone breast tumor imaging using vertical axisof-rotation (VAOR) SPECT system: an initial study, IEEE Conf. Rec. (1997).

[17] S.D. Metzler, J.E. Bowsher, M.P. Tornai, B.C. Pieper, J. Peter, R.J. Jaszczak, SPECT Breast imaging combining horizontal and vertical axes of rotation, IEEE Trans. Nucl. Sci. 49 (1) (2002) 31.

[18] http://WWW.naviscanpet.com.

[19] A. Karimian, C.J. Thompson, S. Sarkar, G. Raisali, R. Pani, H. Davilu, D. Sardari, A dedicated PET system for breast imaging (CYBPET), M2-1, IEEE NSS-MIC Conference Record, Rome, Italy, 2004.

[20] A.F. Chatziioannou, Molecular imaging of small animals with dedicated PET tomographs, Eur. J. Nucl. Med. 29 (1) (2002) 98 .

[21] T.R. DeGrado, T.G. Turkington, J.J. Williams, C.W. Stearns, J.M. Hoffman, R.E. Coleman, Performance characteristics of a whole-body PET scanner, J. Nucl. Med. 35 (8) (1994) 1398.

[22] C.J. Thompson, J. Moreno-Cantú, Y. Picard, PETSIM: Monte Carlo simulation of all sensitivity and resolution parameters of positron imaging systems, Phys. Med. Biol. 37 (3) (1992) 731.

[23] C.J. Thompson, Y. Picard, PETSIM Monte Carlo Simulation Programs Guide to Writing Batch Processing Command Files. Revision 2003.

[24] R.R. Raylman, S. Majewski, M.F. Smith, R. Wojcik, A.G. Weisenberger, B. Kross, V. Popov, J.J. Derakhshan, 
Comparison of scintillators for positron emission mammography (PEM) systems, IEEE Trans. Nucl. Sci. 50 (1) (2003) 42.

[25] W.W. Moses, Time of flight in PET revisited, IEEE Trans. Nucl. Sci. 50 (5) (2003) 1325.

[26] D.A. Mankoff, L.K. Dunnwald, J.R. Gralow, G.K. Ellis, A. Charlop, T.J. Lawton, et al., Blood flow and metabolism in locally advanced breast cancer: relation to response to therapy, J. Nucl. Med. 43 (4) (2002) 500.

[27] K.R. Zasadny, M. Tatsumi, R.L. Wahl, FDG metabolism and uptake versus blood flow in women with untreated primary breast cancer, Eur. J. Nucl. Med. 30 (2) (2003) 274.

[28] W.W. Moses, J. Qi, Fundamental limits of positron emission mammography, Nucl. Instr. and Meth. Phys. Res. A 497 (2003) 82. 